\title{
Call for Papers: Adenosine in the Central Nervous System
}

\author{
Editor-in-Chief: Sergi Ferré, MD, PhD; Integrative Neurobiology Section, National Institute on Drug Abuse
}

The Journal of Caffeine and Adenosine Research has extended its scope beyond caffeine research to include original research related to adenosine and adenosine receptors in physiology and disease. In the central nervous system, adenosine is a ubiquitous neuromodulator involved in many physiological functions. While caffeine is an adenosine receptor antagonist, adenosine is the endogenous agonist which promotes sleep homeostasis and is actively released following cellular insult as a distress signal that modulates tissue damage and repair, a role that extends beyond the central nervous system. Adenosine and ATP are important messengers in neuronal and glial interactions and function. The role of adenosine and purines has been well established in neurodegeneration, cognition, and psychiatric disorders. Journal of Caffeine and Adenosine Research aims to showcase novel original adenosine research from around the world.

The journal is specifically looking for original research investigating the

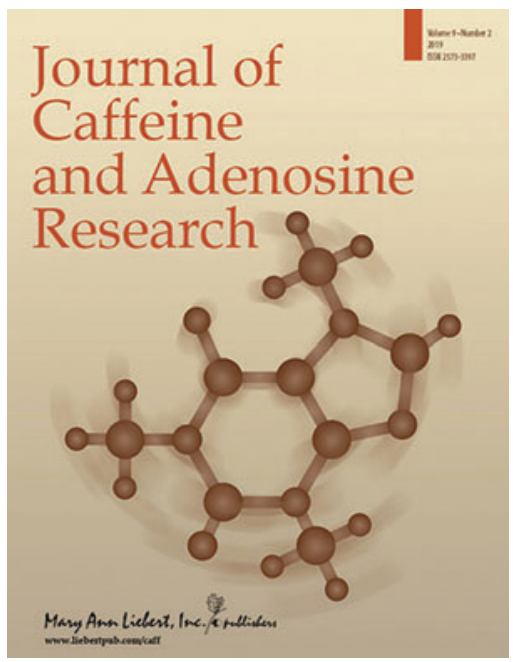
role of adenosine in central nervous system physiology and pathogenesis. We will consider manuscripts in the following categories:

- Adenosine in neurophysiology

- Adenosine in epilepsy and movement disorders

- Adenosine in cognition and neurodegenerative disorders

- Adenosine in psychiatric disorders

- Adenosine in sleep and sleep disorders

- Adenosine in addiction physiology

- Adenosine and the circadian clock in addiction and alcohol use

Advantages of publishing in the Journal of Caffeine and Adenosine Research include:

- Fast and user-friendly electronic submission

- Rapid, high-quality peer review

- Maximum exposure: accessible in 170 countries worldwide

- Open Access options available

Please address any questions to Managing Editor Dr. Karen Cloud-Hansen (kch.editor@gmail.com) and our Editor-in-Chief Dr. Sergi Ferré (sferre@ intra.nida.nih.gov).

\section{Submit your paper for peer review online:} https://mc.manuscriptcentral.com/caffeine 